\title{
Pionic Degrees of Freedom in Atomic Nuclei and Quasielastic Knockout of Pions by High-Energy Electrons
}

\author{
V.G. Neudatchin, N.P. Yudin, L.L. Sviridova, S.N.Yudin \\ Institute of Nuclear Physics, Moscow State University 119899 Moscow, Russia
}

\begin{abstract}
The nonlinear model of pionic condensate in nuclei by G. Preparata can be efficiently verified by investigation of the quasielastic knockout process $A\left(e, e^{\prime} \pi^{ \pm}\right) A^{*}$. First, a momentum distribution (MD) of the collective pions has a bright maximum at $q_{0} \simeq 0.3 \mathrm{GeV}$. Second, the excitation spectrum of a recoil nucleus is concentrated at low energies $E_{r e c, A} \simeq q_{0}^{2} / 2 A m_{N} \leq 1 \mathrm{MeV}$. The results for the pion knockout from mesonic clouds of individual nucleons are absolutely different. The latter results are presented both for pion and $\rho$-meson clouds localized on nucleons.
\end{abstract}

\section{Introduction}

For the last decade the problem of mesons (in particular, pions) in cold and hot nuclei has been one of the most urgent problems of nuclear and hadron physics [1, 2, 3]. Is the number of pions per nucleon in nuclei bigger than in a free nucleon? Do the properties of mesons in nuclei change as compared with ones in vacuum? Do pions give essential additional contribution to the nuclear quark condensate? This is not a full list of urgent questions.

One of the most interesting questions in this field is the question about a possibility of separating pions from nucleons in nuclei and pions forming a collective degree of freedom. In the 70's, A.B. Migdal offered a concept of a pion condensated state in nuclei [4] (for details, see the monograph [5]). Here, three coupled channels in nucleus are considered: a nucleon on an excited shell-model orbital and a nucleon hole, $\Delta$-isobare and a nucleon hole and pions. According to later estimates [6], the real nucleon density in nuclei is less than critical one, which is necessary to form a condensate. In the 90's, G. Preparata with his colleagues [7] proposed a new mechanism of pionic injecting into nuclei. He followed ideas by R. Dicke about the supperradiation in a system of many identical atoms [8], i.e. about the coherent photon emission. The power of the emission in this system can be $N^{2} / 4$ times stronger than that in an isolated atom ( $N$ is the number of atoms). It means that a "superstrong" interaction of a photon field with the system of atoms appears.

G.Preparata extended this concept to a system of nucleons, where $N$ and $\Delta$-isobare play the role of the ground state and an excited state of an atom, respectively, while the

*e-mail:yudin@helene.sinp.msu.ru 
pion field is analogous to the photon one. According to the estimates by G. Preparata, in spite of the large energy losses $(\sim 300 \mathrm{MeV})$, which are necessary to excite the $\Delta$-isobare, a powerful coherent interaction between $N$-, $\pi$ - and $\Delta$ subsystems results in pion condensation in nuclei even at the real density $\rho_{\text {nucl. }}$. This is accompanied by the remarkable increase of the binding energy of the nucleus (which is equal to $\sim 60 \mathrm{MeV}$ per nucleon). The coherence domain includes approximately 70 nucleons. This "superradiative" pionic mode is characterized by momenta of pions $\vec{q}_{0}$,

$$
\sqrt{\vec{q}_{0}^{2}+m_{\pi}^{2}}=m_{\Delta}-m_{N}, \quad q_{0} \simeq 0.3 \mathrm{GeV}
$$

$m_{\Delta}$ and $m_{N}$ are masses of $\Delta$ and $N$.

The number of the collective pions $n_{i, c o l l}$ of each kind $i\left(\pi^{+}, \pi^{-}\right.$or $\left.\pi^{0}\right)$ per nucleon in nuclei with $N=Z$ is approximately 0.1 [7]. It is larger than the corresponding amount of pions in the cloud of an individual nucleon having momenta within $k=(1 \pm 0.1) q_{0}$ range (see below, we mean the $P$-state of the pion in the channel of virtual decay $p \rightarrow n+\pi^{+}$ [9, 10).

The experimental situation with pions in nuclei is contradictory. For a long time it was thought that in nuclei there is a definite deficit of pions. In any case, the number of pions in nuclei is smaller that the RPA approach predicts. This was reflected in the title of the wellknown paper "Where are the nuclear pions ?" [11]. Recently, however an enhancement of pionic degrees of freedom predicted by RPA was observed experimentally. Namely, the longitudinal nuclear response $R_{L}$ in the $(\vec{p} \vec{n})$-reaction on ${ }^{12} C,{ }^{40} \mathrm{Ca},{ }^{28} \mathrm{~Pb}$ nuclei was measured [12]. This reaction is characterized by transfer of pion quantum numbers from proton to the nucleus. Values of $R_{L}$ at the momentum transfer $q=1.7 \mathrm{fm}^{-1}$ and energy transfer $\omega$ around $60 \mathrm{MeV}$ indicate derectly the pionic degrees of freedom in nuclei (for the ${ }^{12} \mathrm{C}$ target the experiment showed an interesting collective enhancement, indicating excitation of the corresponding charged giant resonance [12]). Now, the interest in the problem of pion content of nuclei revives. It is necessary to point out that "Preparata pions" could not probably be observed as an additional longitudinal responce in $(p, n)$ reactions. It is related to an important role of the $\Delta$-isobares in the hypothetical Preparata phenomenon. In the usual consideration of the relation between nucleon particle-hole responce and contents of pions the role of $\Delta$-isobares is not taken into account.

The main aim of the present work is to propose a very independent kind of experiment, which gives us a possibility to see "Preparata pions", i.e.to see directly a momentum distribution (MD, the square of a wave function in the momentum representation) of collective pions, which are not localized on the nucleon. We mean the reaction of quasielastic knockout (QEK) of pions from the nucleus by electrons with energy of a few $\mathrm{GeV}$.

The background of QEK in microphysics is very rich. The exclusive processes of the quasielastic knockout of protons from the atomic nucleus by protons $(p, 2 p)$ or by electrons $(e, e p)$ at bombarding energies of a few hundred $\mathrm{MeV}$ are well known [13]. they were used for investigations of the MDs of nucleons on separated shell-model orbitals. The shape of the MD for light nuclei was proved out to be very sensitive to values of the nucleon shell-model quantum numbers $n l$.

Experimentally, the coincidence technique is used with the energy resolution $\Delta E \sim 1$ $\mathrm{MeV}$, and the interpretation of results is based on a very simple binary conservation laws

$$
E_{0}=E_{1}+E_{2}+E_{b i n d}, \quad \vec{p}_{0}+\vec{q}=\vec{p}_{1}+\vec{p}_{2}
$$


which are valid here, because energies of both initial bombarding particles and two final particles are high (the impulse approximation [14]). In Eq.2, $\vec{q}$ is the initial momentum of a virtual particle to be knocked out, and the rest of the notations is evident. The kinematics of the above process corresponds to the inequalities $|\vec{q}| \ll\left|\vec{p}_{0}\right|,\left|\vec{p}_{1}\right|,\left|\vec{p}_{2}\right|$ and $E_{\text {bind }} \ll E_{0}, E_{1}, E_{2}$. In this way, the values of $\vec{q}$ and $E_{\text {bind }}$ are obtained from the experiment.

One more nuclear example is exclusive reactions $(p, p \alpha)$, etc. of the quasielastic knockout of nucleon clusters by protons with the energy $0.5-1 \mathrm{GeV}$. This reaction can be important for identification at high energies [15] the mechanisms of deexcitation of virtually excited clusters in nuclei.

The QEK process $(e, 2 e)$ at the beam energies of around $10 \mathrm{KeV}$ with the analogous extraction of the electronic MDs is widely used in investigations of the electronic structure of atoms, molecules and solids [16, 17]. So, there is a great experience in the investigation of the exclusive QEK reactions. In our previous papers [9, 10, 18] we have extended the concept of the QEK to the knockout of mesons from nucleons by high energy electrons. It demanded a relativistic generalization of the theory. Namely, the pole $z$-diagram reflecting a virtual creation of, say, $\pi^{+} \pi^{-}$pair was taken into account in addition to the usual diagram of pion knockout (the instantaneous form of dynamics). The second important point was that it is possible to separate experimentally the reactions induced by longitudinal virtual photons $\gamma_{L}^{*}$ and ones induced by transverse virtual photons $\gamma_{T}^{*}$ [19]. This offers a unique way 20] to investigate the MDs of pions $\left(\pi^{+*}+\gamma_{L}^{*} \rightarrow \pi^{+}\right.$subprocess) and $\rho$-mesons $\left(\rho^{+*}+\gamma_{T}^{*} \rightarrow \pi^{+}\right.$subprocess $)$separately, by means of the $p\left(e, e^{\prime} \pi^{+}\right) n$ reaction with the squared mass of virtual photon $Q^{2}$ of about $2-4(\mathrm{GeV} / c)^{2}$.

In the present paper we extend this approach to the investigation of the pionic degrees of freedom in nuclei. The paper is organized as follows. In the second section we represent shortly a relativistic formalism for the QEK reactions. In the third section, a simple expression for the MDs of the collective pion in the Preparata model is derived and compared with that of pions localized on nucleons in a nucleus. In the fourth section, another variable, which is observable in the $\left(e, e^{\prime} \pi\right)$ experiment, the MD of $\rho$-mesons in the nucleus is calculated within the model of $\rho$-meson localization on the nucleons in a nucleus. The fifth section outlines the advantage of the $\left(e, e^{\prime} \pi\right)$ process in comparison with a $(\gamma, \pi)$ reaction and $(\pi, 2 \pi)$ QEK process.

\section{Formalism}

According to the general theory of meson electroproduction [21], the differential cross section of the reaction $T+e \rightarrow R+\pi^{+}+e^{\prime}$ may be separated into longitudinal (L) and transverse $(\mathrm{T})$ components, along with interference terms by varying kinematical variables of the final detected particles, $\pi^{+}$and $e^{\prime}$ (Rosenbluth separation). This important result is usually discussed in terms of the independent variables $Q^{2}=-q^{2}$ ( $q$ is a 4 -momentum of the virtual photon), $W^{2}=\left(p_{\pi}+p_{B}\right)^{2}$ ( $W$ is the invariant mass of the final hadrons), $t=\left(p_{B}-p_{p}\right)^{2}$ [19, 21]. Such a parametrisation is convenient, if we have in mind a broad kinematical region, including, say, resonances. But in a narrow region corresponding to the quasielastik knockout, the traditional five variables $E_{e}^{\prime}, \Omega_{e^{\prime}}, \Omega_{\pi}$ of the nonrelativistic QEK theory are more efficient [9, 10].

Having in mind this remark, we can write for the longitudinal cross section of the 
QEK process on a nucleus an expression, that is very close to formulae in [9, 10]

$$
\frac{d^{5} \sigma_{L}\left(e T \rightarrow e^{\prime} \pi R\right)}{d E_{e^{\prime}} d \Omega_{e^{\prime}} d \Omega_{\pi}}=\frac{E_{e^{\prime}}^{2}}{E_{e}} \frac{I(e \pi)}{E_{\pi}\left(\overrightarrow{k^{\prime}}\right)} 4 \overline{\left|\Psi_{T}^{R \pi}(\vec{k})\right|^{2}} \frac{d \sigma\left(e \pi \rightarrow e^{\prime} \pi\right)}{d \Omega_{\pi}},
$$

where the right part contains only one, predominant pole component [10, 12, 18 at $Q^{2} \approx 2-3(\mathrm{GeV} / c)^{2}$. Here, $E_{e^{\prime}}$ and $\Omega_{e^{\prime}}$ are energy and a solid angle of the final electron; $\mathrm{d} \Omega_{\pi}$ is an element of a solid angle of the final pion in the lab system; $E_{e}$ is energy of the initial electron, $\vec{k}$ is momentum of the virtual pion; $E_{\pi}\left(\vec{k}^{\prime}\right)=\sqrt{\vec{k}^{\prime 2}+m_{\pi}^{2}} ; \overrightarrow{k^{\prime}}$ and $E_{\pi}\left(\overrightarrow{k^{\prime}}\right)$ are momentum and energy of the knocked-out real pion; $I(e \pi)$ is the invariant flux of electrons and pions; $\overline{\left|\Psi_{T}^{R \pi}(\vec{k})\right|^{2}}$ is the MD of pions in the channel $T \rightarrow R \pi$; and, finally, $d \sigma\left(e \pi \rightarrow e^{\prime} \pi\right) / d \Omega_{\pi}$ is a cross section of a free scattering process $e+\pi^{+} \rightarrow e^{\prime}+\pi^{\prime+}$. The bar means spin projection average quantity.

The transverse cross section is given by a formula very close to the formulae in [10, 18]

$$
\frac{d^{5} \sigma_{T}}{d E_{e^{\prime}} d \Omega_{e^{\prime}} d \Omega_{\pi}}=\frac{E_{e^{\prime}}^{2}}{E_{e}} \frac{I(e \rho)}{E_{\rho}(\vec{k})} \overline{\left|\Psi_{p}^{B \rho}(\vec{k})\right|^{2}} \frac{d \sigma\left(e \rho \rightarrow e^{\prime} \pi\right)}{d \Omega_{\pi}} .
$$

Here $E_{\rho}(\vec{k})=\sqrt{\vec{k}^{2}+m_{\rho}^{2}}$. Eq. $(4)$ is valid at $Q^{2}>2(\mathrm{GeV} / c)^{2}$ [18].

Both free cross sections include relevant electromagnetic form factors [10, 18.

The differential cross sections $d \sigma / d \Omega_{\pi}$ for the both mechanisms are given by

$$
\frac{d \sigma}{d \Omega_{\pi}}=\frac{\overline{\left|M_{f i}\right|^{2}}}{64 \pi^{2}} \frac{\left|\overrightarrow{k^{\prime}}\right|}{m_{\pi, \rho} E_{e}^{2}} \frac{1}{1-\left(E_{\pi}\left(\overrightarrow{k^{\prime}}\right) /\left|\vec{k}^{\prime}\right|\right) \cos \theta_{\pi^{\prime} e}}
$$

where $\theta_{\pi^{\prime} e}$ is an angle between the incident electron and the final pion.

For the elastic scattering off point pions the square amplitude is equal to

$$
\overline{\left|M_{f i}\right|^{2}}=64 \pi^{2} \frac{m_{\pi} E_{e}}{E_{e^{\prime}}} \sigma_{M}
$$

for the scattering off point $\rho$-mesons (with de-excitation) (see Appendix)

$$
\overline{\left|M_{f i}\right|^{2}}=64 \pi^{2} \frac{g_{\rho \pi \gamma}^{2}}{m_{\pi}^{2}} \frac{E_{e}}{E_{e^{\prime}}} \sigma_{M}\left[\operatorname{tg}^{2}(\theta / 2)\left((l k)(k q)-3 / 4 m_{\rho}^{2} Q^{2}\right)-1 / 2(k q)^{2}\right],
$$

where $g_{\rho \pi \gamma}$ is the constant of conversion of the $\rho$-meson into pion, $\sigma_{M}$ is the Mott cross section $\sigma_{M}=\left(4 \alpha^{2} \cos ^{2}(\theta / 2) E_{e^{\prime}}^{2}\right) / Q^{4}, \theta$ is an angle between the incident and scattered electrons, $l, k, q$ are 4-momenta of the incident electron, $\rho$-meson and photon.

\section{Momentum Distributions of delocalized pions in nuclei}

Having in mind the Preparata model [7], we suppose for simplicity, that the coherence domain coresponds to the whole nucleus. Hence, a radial wave function of the collective pion has a form of the standing $P$-wave [7]

$$
\begin{gathered}
\Phi(r)=c j_{1}\left(q_{0} r\right), \quad r \leq R, \\
\Phi(r)=0, \quad r>R,
\end{gathered}
$$


and nucleons in the initial and final nuclear states occupy the same shell in the mshellmodel, transitions like $0^{+} \rightarrow 1^{+}, 1^{+} \rightarrow 1^{+}$, etc. take place. The situation is close to weak coupling of the pion to the nucleus. In Eq. (8), $R$ means the radius of the domain. Its value should correspond to $A \simeq 70$ at $N=Z$. Next, the constant $c$ is defined by the normalization of the wave function (8) to the abovementioned value $n_{i, c o l l}=0.1$, so $c=0.027$. By the Fourier transformation of the single-particle wave function (8), we obtain the MD of the collective pions $\left|\Psi_{A}^{A \pi}(\vec{k})\right|^{2}$. This MD corresponds to the longitudinal cross section (3), (5) and is presented in Fig.1 (solid curve) with normalization to one nucleon to facilitate its comparison with the dashed curve (the MD of pions in a free nucleon, see below). The solid curve should be multiplied by $A$ to compare it with experimental data. It has a bright maximum at $k=q_{0}$, i.e. it is close to the plane wave within the limitation imposed by the finite volume of the nucleus. The dashed curve corresponds to the MD of the pion in a free nucleon $\overline{\left|\Psi_{N}^{N \pi}(\vec{k})\right|^{2}}(N \rightarrow N+\pi$ channel of the virtual decay), which we have reconstructed [9, 10] from the $p\left(e, e^{\prime} \pi\right) n$ experiment [19. This experiment, in fact, was carried out at the quasielastic kinematics [9, 10, 18] $\left(Q^{2}\right.$ was large enough: $\left.Q^{2}=1-3(\mathrm{GeV} / c)^{2}\right)$. Our analysis [9, 10, 18] was based on the relativistic pole approximation in the laboratory system, which included a pole $z$-diagram.

Fig.2 represents a washed-out MD of the localized pions $\overline{\overline{\left|\Psi_{N}^{N \pi}(\vec{k})\right|^{2}}}$ (thick solid line) obtained by the convolution of the MD of pions in a free nucleon (dashed line) with the averaged MD of the shell-model nucleons in nucleus $\overline{\left|\Phi_{A}^{A-1, N}(\vec{p})\right|^{2}}$ (solid line):

$$
\overline{\overline{\left|\Psi_{N}^{N \pi}(\vec{k})\right|^{2}}}=\int \overline{\left|\Psi_{N}^{N \pi}\left(\vec{k}+\left(m_{\pi} / m_{N}\right) \vec{p}\right)\right|^{2}} \cdot \overline{\left|\Phi_{A}^{A-1, N}(\vec{p})\right|^{2}} d \vec{p} .
$$

Both the MD of pions in a free nucleons and the washed-out MD of the localized pions, in contrast to the MD of pions in the Preparata model, are very smooth at $k$ values, which are close to $q_{0}$. The momentum distributions corresponding to Fig.1,2 are isotropic with respect to $\vec{q}$ direction, because they are averaged over the magnetic quantum numbers of the pionic $P$-orbital.

The main point here is that the knockout of the delocalized collective pions is accompanied by recoil to the final nucleus as a whole, with the corresponding very small recoil energy $E_{r e c, A} \simeq q_{0}^{2} / 2 A m_{N}<1 \mathrm{MeV}(A \simeq 70-80)$, although the momentum $q_{0}$ itself is not small (see Eq. 1). This is why such prediction is not trivial. The MD of such pions has a sharp maximum at $k=q_{0}$.

The wave function of pions (8) does not contain pion-nucleon spacious correlations and, as a result, the final recoil nucleus will not be internally excited. In fact, the best experimental energy resolution $\Delta E$ may be around $10 \mathrm{MeV}$ here, and this severe simplification partly softens. The real situation will correspond to the summation over many exited states of the external shell of the final nucleus, i.e. to a sum rule.

At the same time, the opposite extreme case of the knockout of pions with the same virtual momentum $\vec{k}, k \simeq q_{0}$ from the pion cloud of an individual nucleon will be characterized by a large value of the recoil energy transfered to one nucleon, $E_{r e c, N} \simeq q_{0}^{2} / 2 m_{N} \simeq 50$ $\mathrm{MeV}$. This nucleon, with a large probability, will be directly emitted from the nucleus (we mean here the numerous weakly bound nucleons of the external shell). A reliable identification of this event requires triple coincidences $e^{\prime}+\pi^{-}+p$, which is an urgent experimental problem. In a noncomplete experiment with only double coincidences $e^{\prime}+\pi^{-}$, the abovementioned event will be perceived as a corresponding high excitation $(\omega \simeq 50$ 
$\mathrm{MeV}$ ) of the final recoil nucleus accompanied by the transfer of the momentum $-\vec{k}, k \simeq q_{0}$ to this nucleus. This group of events will show a very smooth MD of the virtual pions like the corresponding part of the thick solid line in Fig.2 at $k$ around $q_{0}$. The discussed recoil of the proton in the process of the $\pi^{-}$knockout from the nucleus can also create one of the charged nuclear giant resonances with the excitation energy $\omega$ of a few tens $\mathrm{MeV}$ (see above, the discussion of the $(\vec{p}, \vec{n})$ meson transfer reaction). An intriguing new opportunity here is to investigate a $k$-dependence of such cross sections.

So, the principal result of this section is that the high-energy pion electroproduction on nuclei by means of the virtual longitudinal photons $\gamma_{L}^{*}$ at the kinematics of the QEK process at small $\omega$ values of a few $\mathrm{MeV}$ offers an opportunity to see the cooperative, maximally delocalized pions in nuclei by the most direct way. The bright MD maximum at $k \simeq q_{0} \simeq 0.3 \mathrm{GeV} / c$ (the solid line in Fig.1) will be the principal sign of the presence of such pions in the nucleus. The increase of $\omega$ corresponds, qualitatively, to increasing localization of the discussed virtual pions in the nucleus, and the shape of the MD measured at different $\omega$ may be helpful for the clarification of evolution of the reaction mechanism with the increase of the excitation energy $\omega$ (the usage of the triple coincidence would be very urgent here, too).

It must be noted, that final energy of the knocked-out pion should be not lower than 1 $\mathrm{GeV}$ to avoid disturbing influence of the intermediate resonance $(\Delta$-isobare) in the $\pi-A$ final state interaction. Energies much higher than $1 \mathrm{GeV}$ are also not suitable because they correspond to the different physics of asymptotical quark counting rules [24]. This physics, which corresponds to the $Q^{2}$ values of $10-20(\mathrm{GeV} / \mathrm{c})^{2}$ and rather small cross sections, is very popular now [25]. But our physics of the soft hadronic degrees of freedom in the nucleons and nuclei, which corresponds to rather moderate $Q^{2}$ values of 2-4 $(\mathrm{GeV} / \mathrm{c})^{2}$ and which is unfortunately still in the shadow, is not less interesting. By the way, it corresponds to the quite measurable cross sections.

\section{Momentum distributions of $\rho$-mesons localized on nucleons in nuclei}

As it was noted in the section 1 , here we will discuss the model of $\rho$-mesons localized on nucleons in the nucleus. The MD of the $\rho$-mesons may be obtained by means of the QEK reaction such as $A(N, Z)\left(e, e^{\prime} \pi\right) A(N-1, Z+1 ; \omega)$ initiated by the transverse virtual photons, $\rho^{-}+\gamma_{T}^{*} \rightarrow \pi^{-}$. The principal experimentally observed difference of the model under consideration from the discussed above model of the pionic degrees of freedom is that the MD should be the same for any $\omega$ value, i.e. that the MD does not depend on a microscopic process, which follows the recoil to the nucleon (at the small $k, k \leq 0.1$ $\mathrm{GeV} / c$, either a soft nuclear excitation within the lowest shell-model configuration for small $\omega$ of a few $\mathrm{MeV}$, or an excitation of the charged giant resonance for $\omega \simeq 20-40$ $\mathrm{MeV}$ take place; at the relatively large $k, k \simeq q_{0}$, either an excitation of the charged giant resonance at $\omega \simeq 30 \mathrm{MeV}$ or a direct emission of the recoil nucleon at $\omega \simeq 50-60 \mathrm{MeV}$ take place, etc.).

Starting with the $\rho$-meson MD in the nucleon $\overline{\left|\Psi_{N}^{N \rho}(\vec{k})\right|^{2}}$ (dashed line in Fig.3) 10, 18, we take into account Fermi motion of nucleons in the nucleus by means of Eq.(9) and obtain, finally, the solid curve in Fig.3 analogous to the thick solid curve in Fig.2 but extended to much larger values of $k$ due to the large value of the mass $m_{\rho} \simeq 800 \mathrm{MeV}$. 
This curve represents the washed-out MD of $\rho$-mesons in the nucleus $\overline{\overline{\left|\Psi_{N}^{N \rho}(\vec{k})\right|^{2}}}$ if they are localized on nucleons. A deviation of experimental results from this shape, similar to the deviation of the solid curve in Fig.1 from the thick solid curve in Fig.2 will mean the delocalization of $\rho$-mesons in the nucleus (see above, section 2 ).

\section{Conclusion}

In this paper, we have proposed a program of the direct experimental investigation of the pionic wave functions in nuclei by means of the quasielastic knockout reactions $A(N, Z)\left(e, e^{\prime} \pi^{-}\right) A(N-1, Z+1 ; \omega)$ or $A(N, Z)\left(e, e^{\prime} \pi^{+}\right) A(N+1, Z-1 ; \omega)$ initiated by electrons with energy of a few $\mathrm{GeV}$ and mediated by the longitudinal virtual photons. It has been demonstrated, that the momentum distribution of the delocalized pions in the cooperative model by Preparata is qualitatively different from the MD of the pions localized on nucleons in the nucleus. It is expected that the corresponding spectra of excitation energies of the final nucleus-spectator, $\omega$, will be rather different in these two cases.

Futher, having in mind the $\left(e, e^{\prime} \pi^{-}\right)$or $\left(e, e^{\prime} \pi^{+}\right)$reaction mediated by the transverse photons, we have demonstrated, as a basic example, the MD of $\rho$-mesons in nucleus within the simplest model of $\rho$-mesons localized on nucleons in the nucleus.

Recently, the investigation of the $(\pi, 2 \pi)$ quasielastic knockout process has been initiated [26], although the energies of the knocked-out pions are still not high enough. The cross sections here are larger (strong interaction) than those for the $\left(e, e^{\prime} \pi\right)$ reaction, and the $(\pi, 2 \pi)$ reaction gives an opportunity to investigate the $\pi^{0}$-component of the collective field, but distortion and absorption effects [27] for three pionic waves in the $(\pi, 2 \pi)$ reaction are much more pronounced than those for one pionic wave in the $\left(e, e^{\prime} \pi\right)$ process. So, these two reactions, the $\left(e, e^{\prime} \pi\right)$ reaction of a volume character with smaller cross sections and the $(\pi, 2 \pi)$ reaction of a surface character with larger cross sections, can complement each other rather efficiently.

Finally, it should be noted, that the analogous process $(\gamma, \pi)$ on nuclei $\left(Q^{2}=0\right)$ corresponds to the interference of amplitudes for a few different diagrams [28] and does not offer a direct way for extracting the MDs of pions in nuclei.

The authors are grateful to Profs. A.A. Ogloblin and B.S. Slowinsky for their lively interest in the discussed problems.

This work is supported by the Russian Foundation of Fundamental Research (grant N 00-02-16117). 


\section{Appendix}

We will present here the main steps of calculating the amplitude of the process $e+\rho \rightarrow$ $e^{\prime}+\pi$.

The invariant amplitude $M_{f i}$ for this process is equal to

$$
M_{f i}=\frac{e^{2} g_{\rho \pi \gamma}}{Q^{2} m_{\pi}}\left(\bar{u}^{\prime} \gamma_{\beta} u\right) \varepsilon^{\beta \mu \alpha \nu} e_{m \mu} q_{\alpha} k_{\nu} .
$$

Here $g_{\rho \pi \gamma}$ is the constant of $\rho \pi \gamma$-interaction, $\bar{u}^{\prime}, u$ are the Dirac's spinors of the electron (with the normalization $\bar{u} u=2 m_{e}$ ), $\gamma_{\beta}$ are the Dirac's matrices, $\varepsilon^{\beta \mu \alpha \nu}$ is a an antisimmetrical unity tensor, $e_{m \mu}$ is a vector of polarisation of the $\rho$-meson.

The summarizing over polarizations should be clarified.

$$
\overline{\left|\overline{u^{\prime}} \gamma_{\beta} u \cdot \varepsilon^{\beta \mu \alpha \nu} e_{m \mu} q_{\alpha} k_{\nu}\right|^{2}}=\frac{1}{2} \frac{1}{3} \sum_{m \mu \mu^{\prime}}\left(\overline{u^{\prime}} \gamma_{\beta} u\right)\left(\bar{u}^{\prime} \gamma_{\beta^{\prime}} u\right)^{*} \varepsilon^{\beta \mu \alpha \nu} e_{m \mu} q_{\alpha} k_{\nu} \varepsilon^{\beta^{\prime} \mu^{\prime} \alpha^{\prime} \nu^{\prime}} e_{m \mu^{\prime}}^{*} q_{\alpha^{\prime}} k_{\nu^{\prime}}
$$

Having calculated electron traces, we obtain

$$
\begin{gathered}
\overline{\left|\bar{u}^{\prime} \gamma_{\beta} u \cdot \varepsilon^{\beta \mu \alpha \nu} e_{m \mu} q_{\alpha} k_{\nu}\right|^{2}}=\frac{1}{3} 2\left(2 l_{\beta} l_{\beta^{\prime}}-g_{\beta \beta^{\prime}}\left(l l^{\prime}\right)\right) \varepsilon^{\beta \mu \alpha \nu} \varepsilon^{\beta^{\prime} \mu^{\prime} \alpha^{\prime} \nu^{\prime}}\left(-g_{\mu m u^{\prime}}+\frac{k_{\mu} k_{\mu^{\prime}}}{m_{\rho}^{2}}\right) q_{\alpha} k_{\nu} q_{\alpha^{\prime}} k_{\nu^{\prime}} \\
=\frac{2}{3}\left(2 l_{\beta} l_{\beta^{\prime}} \varepsilon^{\beta \mu \alpha \nu} \varepsilon_{\mu}^{\beta^{\prime} \alpha^{\prime} \nu^{\prime}} q_{\alpha} k_{\nu} q_{\alpha^{\prime}} k_{\nu^{\prime}}-\left(l l^{\prime}\right) \varepsilon^{\beta \mu \alpha \nu} \varepsilon_{\beta \mu}^{\alpha^{\prime} \nu^{\prime}} q_{\alpha} k_{\nu} q_{\alpha^{\prime}} k_{\nu^{\prime}}\right) .
\end{gathered}
$$

The unity tensors are convoluted according to the following rules:

$$
\begin{gathered}
\varepsilon^{\beta \mu \alpha \nu} \varepsilon_{\beta \mu}^{\alpha^{\prime} \nu^{\prime}}==2 \varepsilon_{\alpha^{\prime} \nu^{\prime}}^{\alpha \nu}=2\left[\delta_{\alpha \alpha^{\prime}} \delta_{\nu \nu^{\prime}}-\delta_{\alpha \nu^{\prime}} \delta_{\alpha^{\prime} \nu}\right] \\
\varepsilon^{\beta \mu \alpha \nu} \varepsilon_{\mu}^{\beta^{\prime} \alpha^{\prime} \nu^{\prime}}=\varepsilon_{\beta^{\prime} \alpha^{\prime} \nu^{\prime}}^{\beta \alpha \nu} .
\end{gathered}
$$

Thus, we obtain

$$
\overline{\left|\bar{u}^{\prime} \gamma_{\beta} u \cdot \varepsilon^{\beta \mu \alpha \nu} e_{m \mu} q_{\alpha} k_{\nu}\right|^{2}}=4\left[\left(l l^{\prime}\right)\left(q^{2} k^{2}-(k q)^{2}\right)-2(l k)(l q)(k q)+(l q)^{2} k^{2}+(k l)^{2} q^{2}\right] .
$$

This equation permits us to find easily the cross section (7). 


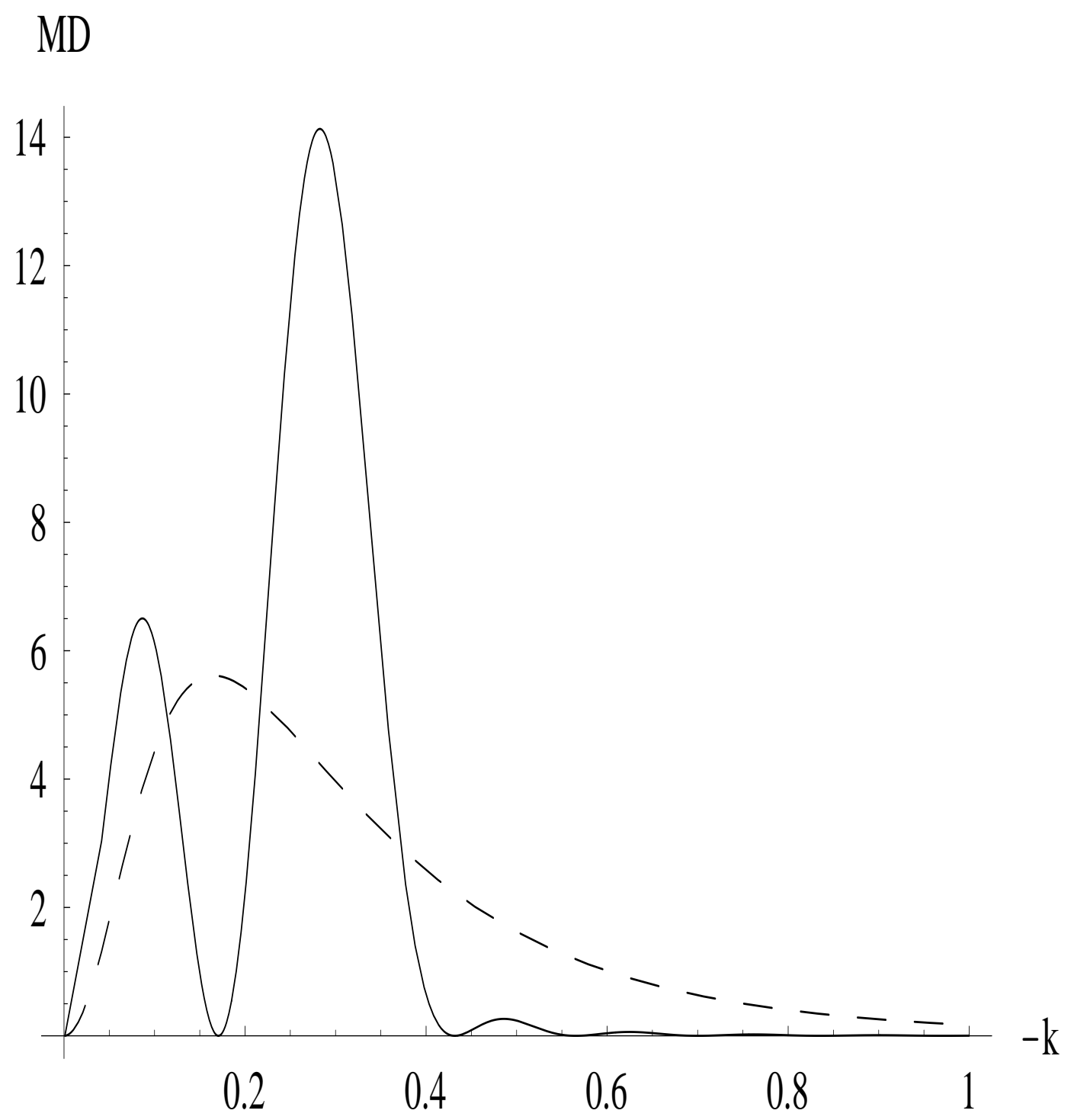

Figure 1: Momentum distribution of pions, versus $|k|, \mathrm{GeV} / \mathrm{c}$ : solid curve - the MD of pions in nuclei, Preparata model $\overline{\left|\Psi_{A}^{A \pi}(\vec{k})\right|^{2}},(\mathrm{GeV} / \mathrm{c})^{-3}$; dashed curve - the MD of pions in a free nucleon $\overline{\left|\Psi_{N}^{N \pi}(\vec{k})\right|^{2}},(\mathrm{GeV} / \mathrm{c})^{-3}$. 


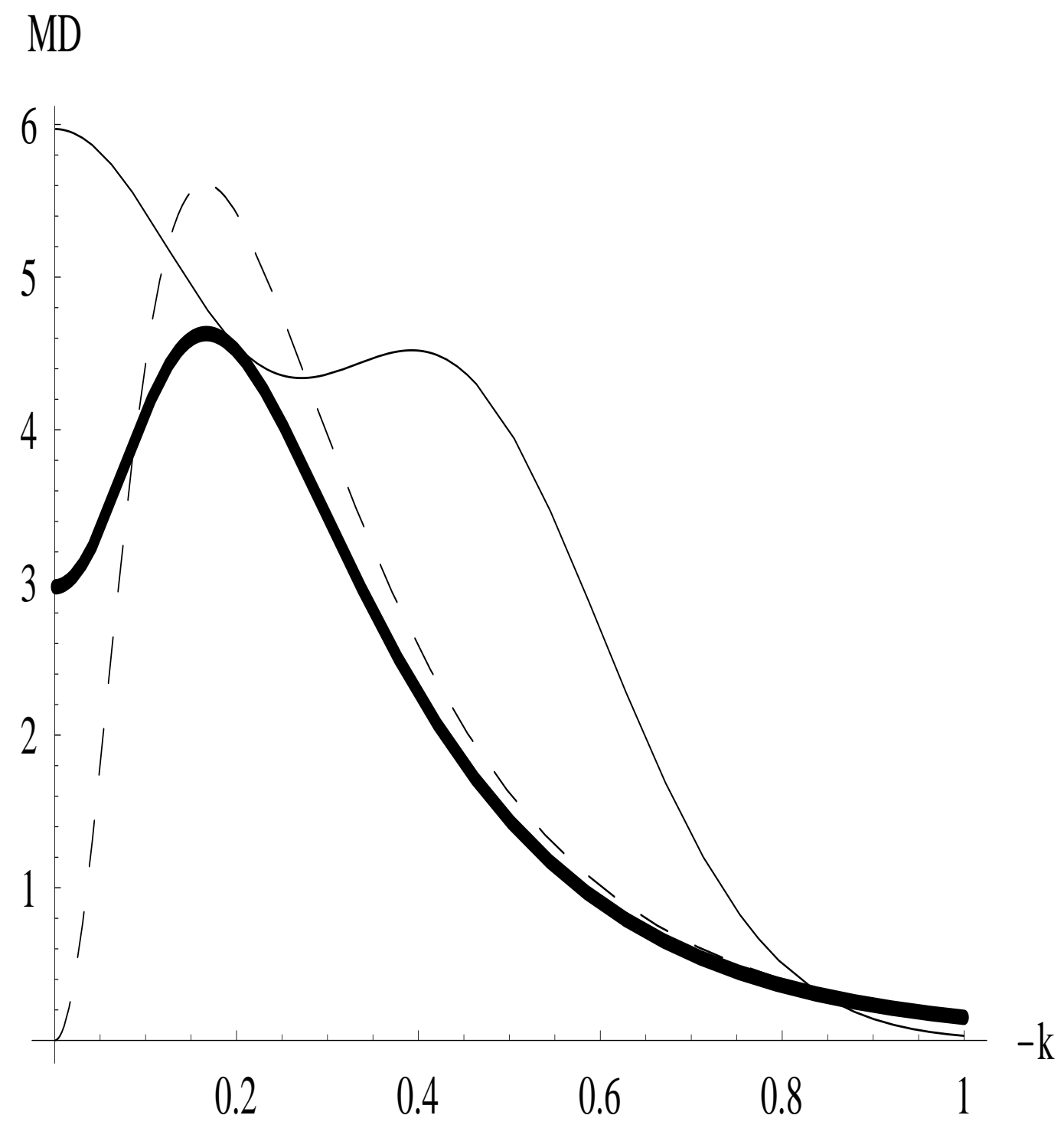

Figure 2: MD of pions in a nucleus versus $|k|, \mathrm{GeV} / \mathrm{c}$. Dashed line is the MD of pions in a free nucleon $\overline{\left|\Psi_{N}^{N \pi}(\vec{k})\right|^{2}},(\mathrm{GeV} / c)^{-3}$. Thin solid line is the average MD of a nucleon in the nucleus $\overline{\left|\Phi_{A}^{A-1, N}(\vec{k})\right|^{2}},(\mathrm{GeV} / c)^{-3}$. Thick solid line is the washed-out MD of localized pions in the nucleus $\overline{\overline{\left|\Psi_{N}^{N \pi}(\vec{k})\right|^{2}}},(\mathrm{GeV} / c)^{-3}$. 


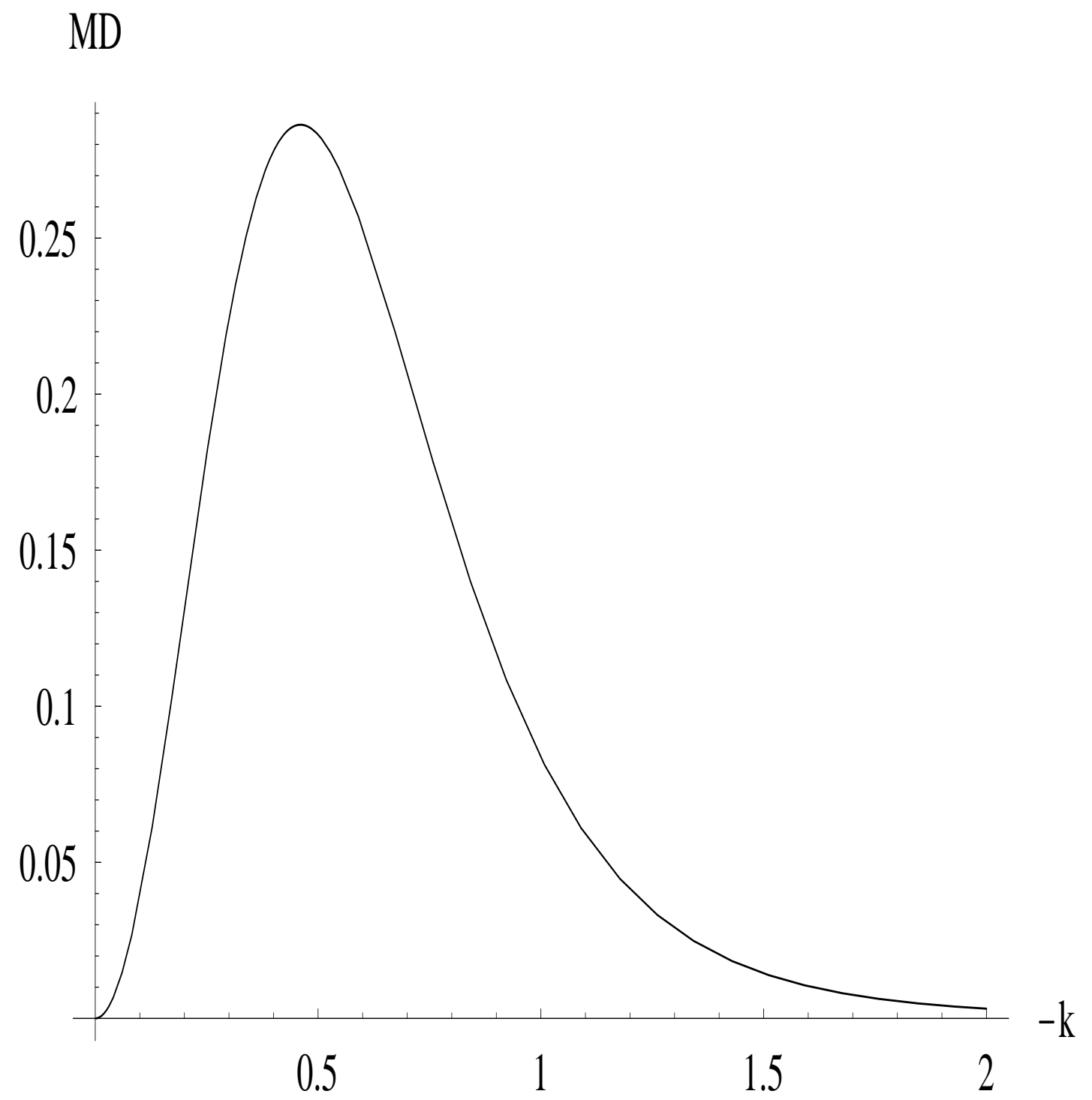

Figure 3: MD of the $\rho$-mesons in a nucleus versus $|k|, \mathrm{GeV} / \mathrm{c}$. Dashed line is the MD of the $\rho$-mesons in a free nucleon $\overline{\left|\Psi_{N}^{N \rho}(\vec{k})\right|^{2}},(\mathrm{GeV} / c)^{-3}$. Solid line is the MD of the $\rho$-mesons in a nucleus $\overline{\overline{\left|\Psi_{N}^{N \rho}(\vec{k})\right|^{2}}},(\mathrm{GeV} / c)^{-3}$. 


\section{References}

[1] E.G. Drukarov, M.G. Ryskon and V.A. Sadovnikova, Prog.Part.Nucl.Phys. 47, 73 (2001)

[2] R. Rapp, J. Wambach, Adv.Nucl.Phys. 25, 1 (2000)

[3] G. Brown and M. Rho, Phys.Rep. 269, 333 (1996); 363, 85 (2002)

[4] A.B. Migdal, Rev.Mod.Phys. 50, 107 (1978)

[5] A.B. Migdal, D.N. Voskresensky, E.E. Saperstein, and M.A. Troitsky, "Pionic Degrees of Freedom in Nuclear Matter" (in Russian), Nauka, Moscow, 1991

[6] Yu.B. Ivanov, J. Knoll, H. VanHees, and D.N. Voskresensky, Yad.Fiz. 64, 711 (2001)

[7] R. Alzetta, G. Liberti, and G. Preparata, Nucl.Phys. A 585, 307c (1995); R. Alzetta, T. Bubba, R. LePerra, et al., Nuovo Cimento A 112, 762 (1999); R. Alzetta, G. Liberti, and G. Preparata, Nuovo Cimento A 112, 1609 (1999)

[8] R.H. Dicke, Phys.Rev. 93, 99 (1954); A.V. Andreev, V.I. Emelianov, and Yu.A. Il'insky, "Coherent Phenomena in Optics" (in Russian), Nauka, Moscow, 1988

[9] V.G. Neudatchin, N.P. Yudin, and L.L. Sviridova, Yad. Fiz. 60, 2020 (1997)

[10] V.G. Neudatchin, L.L. Sviridova, and N.P. Yudin, Yad. Fiz. 64, 1680 (2001)

[11] G.E.Brown, M. Balealla, Z.B. Li and T. Wamack, Nucl.Phys. A 593, 295 (1995); G.F. Bertseh, L. Frankfort and M. Strikman, Science 259, 777 (1993)

[12] H. Sakai, Nucl.Phys. A 690, 66c (2001)

[13] S.S. Volkov, A.A. Vorobiev, O.A. Domchenkov, et al., Yad.Fiz. 52, 1339 (1990); A.A. Vorobiev, Yu.V. Dotsenko, A.A. Lobodenko et al., Yad.Fiz. 58, 3 (1994); D.G. Ireland, and G. Van Der Steenhoven, Phys.Rev. C 49, 2182 (1994); J.Ryckebush, W. Van Nespen, and S. Janssen, Phys.Rev. C 62, 024611 (2000)

[14] M.L. Goldberger and K.M. Watson, "Collision Theory", Wiley, N.Y., 1964

[15] V.G. Neudatchin, A.A. Sakharuk, V.V. Kurovsky, and Yu.M. Tchuvilsky, Phys.Rev. C 50, 148 (1994); Phys.Rev. C 51, 784 (1995)

[16] V.G. Neudatchin, Yu.V. Popov, and Yu.F. Smirnov, Uspekhi Fiz.Nauk 169, 1111 (1999)

[17] E. Weigold and I. McCarthy, "Electron Momentum Spectroscopy", Kluver Academic/Plenum Publishers, N.Y., 1999

[18] N.P. Yudin, L.L. Sviridova, and V.G. Neudatchin, Yad. Fiz. 61, 1689 (1998); 62, 694 $(1999) ; 65, . .(2002)$

[19] C.J. Bebek, C.N. Brown, S.D. Holmes, et al., Phys.Rev. D 17, 1693 (1978); P. Brauel, T. Canzler, D. Cords, et al., Z.Phys. C 3, 101 (1979) 
[20] J. Speth and V. Zoller, Phys.Lett. B 351, 533 (1995)

[21] G. Chanfray, Nucl.Phys. A 429, 489 (1984); V. Dmitrosinivich and F. Gross, Phys.Rev. C 40, 2479 (1989); P.J. Mulders, Phys.Rep. 185, 83 (1990); S. Boffi, C. Guisi, and F.D. Pacati, Phys.Rep. 226, 1 (1993); J.J. Kelly, Phys.Rev. C 60, $054611(1999)$

[22] M. Traini, Phys.Rev. C 55, 1600 (1997); K.I. Blomqvist, W.U. Boeglin, R. Bohm et al., Nucl.Phys. A 626, 871 (1997)

[23] D.O. Riska and G.E. Brown, Nucl.Phys. A 679, 577 (2001)

[24] M. Strikman, Nucl.Phys. A 663, 64c (2000); M. Vanderhaeghen, P.A.M. Guichon, and M. Guidal, Phys.Rev. D 60, 094017 (1999); Nucl.Phys. A 663, 301c (2000)

[25] M. Vanderhaeghen, Eur.Phys.J. A 8, 455 (2000)

[26] F.Bonutti, P. Camerini, E.Fragiacomo, et al. Phys.Rev. C 55, 2999 (1997)

[27] S. Khallaf and A. Ebrahim, Phys.Rev. C 62, 024603 (2000); S. Jena and S. Swain, Phys.Rev. C 55, 3015 (1997); M.H. Cha, Y.J. Kim, Phys.Rev. C 54, 429 (1996)

[28] N.P. Yudin, L.L. Sviridova, and V.G. Neudatchin, Yad. Fiz. 65, 594 (2002) 